\title{
Usefulness of Non-Contrast-Enhanced MR Angiography Using a Silent Scan for Follow-Up after Y-Configuration Stent- Assisted Coil Embolization for Basilar Tip Aneurysms
}

(D) N. Takano, DM. Suzuki, DR. Irie, (D) M. Yamamoto, (D) N. Hamasaki, (DK. Kamagata, (DK.K. Kumamaru, (D) M. Hori, (D) H. Oishi, and (D) S. Aoki

\begin{abstract}
BACKGROUND AND PURPOSE: Y-configuration stent-assisted coil embolization is used for treating wide-neck aneurysms. Noninvasive alternatives to $\mathrm{x}$-ray DSA for follow-up after $\mathrm{Y}$-configuration stent-assisted coil embolization treatment are required. This study aimed to assess the usefulness of non-contrast-enhanced MRA by using a Silent Scan (silent MRA) for follow-up after Y-configuration stent-assisted coil embolization for basilar tip aneurysms.
\end{abstract}

MATERIALS AND METHODS: Seven patients treated with Y-configuration stent-assisted coil embolization for basilar tip aneurysms underwent silent MRA, 3D TOF-MRA, and DSA. Silent MRA and 3D TOF-MRA images were obtained during the same scan session on a 3T MR imaging system. Two neuroradiologists independently reviewed both types of MRA images and subjectively scored the flow in the stents on a scale of 1 (not visible) to 5 (nearly equal to DSA) by referring to the latest DSA image as a criterion standard. Furthermore, we evaluated the visualization of the neck remnant.

RESULTS: In all patients, the 2 observers gave a higher score for the flow in the stents on silent MRA than on 3D TOF-MRA. The average score \pm standard deviation was $4.07 \pm 0.70$ for silent MRA and $1.93 \pm 0.80(P<.05)$ for 3D TOF-MRA. Neck remnants were depicted by DSA in 5 patients. In silent MRA, neck remnants were depicted in 5 patients, and visualization was similar to DSA; however, in 3D TOF-MRA, neck remnants were depicted in only 1 patient.

CONCLUSIONS: Silent MRA might be useful for follow-up after Y-configuration stent-assisted coil embolization.

ABBREVIATIONS: $\mathrm{BA}=$ basilar artery; $\mathrm{CE}-\mathrm{MRA}=$ contrast-enhanced MRA; $\mathrm{PCA}=$ posterior cerebral artery; $\mathrm{UTE}=$ ultrashort echo time

n recent years, intracranial stents have been used for the treatment of wide-neck aneurysms. The Y-configuration stent-assisted coil embolization technique has been generally used for wide-neck bifurcation aneurysms such as those at the tip of the

Received June 29, 2016; accepted after revision October 12.

From the Department of Radiology (N.T., M.S., K. Kamagata, K.K. Kumamaru, M.H., S.A.), Graduate School of Medicine, Juntendo University, Tokyo, Japan; Department of Radiology (N.T., M.S., R.I., N.H., K. Kamagata, K.K. Kumamaru, M.H., S.A.), Juntendo University Hospital, Tokyo, Japan; and Departments of Neurosurgery (M.Y., H.O.) and Neuroendovascular Therapy (H.O.), Juntendo University School of Medicine, Tokyo, Japan.

This work was supported in part by the ImPACT Program of Council for Science, Technology and Innovation (Cabinet Office, Government of Japan) and by a Grantin-Aid for Scientific Research on Innovative Areas (ABiS) from the Ministry of Education, Culture, Sports, Science and Technology of Japan.

Paper previously presented as an electronic poster at: European Congress of Radiology, March 2-6, 2016; Vienna, Austria. doi:10.1594/ecr2016/C-1028.

Please address correspondence to Nao Takano, MMSc, Department of Radiology, Graduate School of Medicine, Juntendo University, 2-1-1 Hongo, Bunkyo-ku, Tokyo 113-8421, Japan; e-mail: ntakano@juntendo.ac.jp

-- Indicates open access to non-subscribers at www.ajnr.org

$\equiv$ Indicates article with supplemental on-line table.

http://dx.doi.org/10.3174/ajnr.A5033 basilar artery (BA). ${ }^{1-6}$ The Y stent deploys 2 stents from the BA to the bilateral posterior cerebral artery (PCA). The 2 stents overlap in the distal BA trunk, with 1 stent penetrating the mesh of the other. The use of 2 stents in a "Y" configuration to assist with coil embolization for bifurcation aneurysms has been accepted for broad-neck aneurysms.

$\mathrm{X}$-ray DSA is the standard technique used for follow-up after an intracranial stent. However, DSA is an invasive technique that carries a risk of neurologic complications, contrast materials, and $\mathrm{x}$-ray radiation. ${ }^{7-10}$

On the other hand, 3D TOF-MRA is widely used as a noninvasive substitute for DSA for the follow-up of coiled aneurysms. ${ }^{11-14}$ Although there have been reports of 3D TOF-MRA being used after stent-assisted coil embolization, ${ }^{12,13}$ it remains difficult to visualize flow in an intracranial stent when using this method because of magnetic susceptibility and radiofrequency shielding. Therefore, contrast-enhanced MRA (CE-MRA) is used for follow-up after stent-assisted coil embolization. However, the use of contrast materials in CE-MRA is associated with nephrogenic systemic fibrosis and anaphylactic shock; therefore, this 
technique might not be appropriate for repeated examinations. ${ }^{15-17}$ Furthermore, it has been reported that gadoliniumbased contrast material accumulates in the dentate nucleus and globus pallidus. ${ }^{18}$

Silent MRA uses a Silenz pulse sequence (GE Healthcare, Milwaukee, Wisconsin) containing an ultrashort echo time (UTE) combined with arterial spin-labeling. Data acquisition is based on $3 \mathrm{D}$ radial sampling, and the arterial spin-labeling technique is used as a preparation pulse for visualization of the blood flow. ${ }^{19,20}$ It is a non-contrast-enhanced MRA technique; therefore, it is better for the patient and suitable for repeated follow-ups.

UTE of silent MRA minimizes phase dispersion of the labeled blood flow signal and decreases magnetic susceptibility to coils and stents. Thus, silent MRA can evaluate the blood flow in an intracranial stent. ${ }^{20}$

To the best of our knowledge, there have been no studies of the use of non-contrast-enhanced MRA for follow-up after Y-configuration stent-assisted coil embolization for basilar tip aneurysms. Therefore, in the present study, we evaluated the usefulness of silent MRA compared with 3D TOF-MRA for follow-up after Y-configuration stent-assisted coil embolization for basilar tip aneurysms.

\section{MATERIALS AND METHODS}

Between October 2014 and September 2015, 7 aneurysm cases treated with Y-configuration stent-assisted coil embolization were retrospectively examined. Written informed consent was not required because of the retrospective nature of this study. All patients had incidentally found unruptured aneurysms and were followed-up with silent MRA, 3D TOF-MRA, and DSA. The average interval between the latest DSA and both MRAs was 27 days (range, 1-180 days). The average interval between the aneurysm treatment and both MRAs was 187.9 days (range, 1-395 days).

The DSA of catheter-based intraarterial cerebral angiography was performed with the following biplane angiographic systems: the AXIOM Artis dTA (Siemens, Erlangen, Germany) until February 2014 and the Artis Q BA Twin (Siemens) from March 2014 to present.

The intracranial stents used were the Enterprise stent (Codman \& Shurtleff, Raynham, Massachusetts), Neuroform stent (Stryker Neurovascular, Kalamazoo, Michigan), and LVIS (Lowprofile Visualized Intraluminal Support) Jr stent (MicroVention, Tustin, California).

Silent MRA and 3D TOF-MRA were performed during the same scan session on a 3T MR imaging system (Discovery MR750w; GE Healthcare) with a 12-channel head-neck coil. The scan parameters of silent MRA were as follows: TR, $1116.4 \mathrm{~ms}$; TE, $0.016 \mathrm{~ms}$; flip angle, $5^{\circ}$; FOV , $180 \times 180 \mathrm{~mm}$; matrix, $150 \times 150$; section thickness, $1.2 \mathrm{~mm}$; NEX, 1.5; bandwidth, $\pm 20 \mathrm{kHz}$; and acquisition time, 7 minutes, 40 seconds. The scan parameters of 3D TOF-MRA were as follows: TR, $19 \mathrm{~ms}$; TE, $2.9 \mathrm{~ms}$; flip angle, $15^{\circ}$; FOV, $200 \times 200 \mathrm{~mm}$; matrix, $416 \times 192$; section thickness, $1.2 \mathrm{~mm}$; NEX, 1; bandwidth, $\pm 41.7 \mathrm{kHz}$; and acquisition time, 3 minutes, 31 seconds ( 3 slabs, overlap between a slab: 10 sections, 1 slab: 32 sections)

Visualization of the parent artery with silent MRA and 3D TOF-MRA was compared with that of the DSA images. The latest
DSA images were used as a criterion standard. Silent MRA and 3D TOF-MRA images were processed to MIP in the same manner as the DSA images. We evaluated DSA images and 2 types of MRA images. The 2 types of MRA images were independently assessed to minimize bias from the knowledge of the results of the other MRA image. Two experienced neuroradiologists (M.S., R.I.) independently reviewed silent MRA and 3D TOF-MRA and rated the conditions of visualization of the flow in each stent subjectively on a 5-point scale as follows: 1 , not visible (almost no signal in the stent); 2 , poor (structures are slightly visible, but with substantial blurring or artifacts); 3, acceptable (acceptable quality diagnostic information with medium blurring or artifacts); 4, good (good quality diagnostic information with minimum blurring or artifacts); or 5, excellent (the depiction is almost equal to DSA). This scale is used to assess the image quality. Furthermore, we evaluated the visualization of the neck remnants of silent MRA and 3D TOF-MRA in patients in whom DSA indicated neck remnants. The scores given by the 2 observers were averaged, and a Wilcoxon signed rank test was performed in the statistical analysis of the subjective scores for the flow in the stents. $P<.05$ was considered statistically significant.

\section{RESULTS}

Patient data and the scores given by the 2 observers are shown in the On-line Table. Both observers gave silent MRA a higher score than 3D TOF-MRA in all cases. The average score \pm standard deviation was $4.07 \pm 0.70$ for silent MRA and $1.93 \pm 0.80$ for $3 \mathrm{D}$ TOF-MRA $(P<.05)$. Neck remnants were depicted by DSA in 5 cases (case number 1, 3, 4, 5, and 7). In silent MRA, neck remnants were depicted in all of these 5 cases, and visualization was similar to DSA; however, in 3D TOF-MRA, neck remnants were depicted in only 1 case. Figs $1-3$ show the silent MRA, 3D TOF-MRA, and DSA images for each case.

\section{DISCUSSION}

The Y-configuration stent-assisted coil embolization technique has been used for wide-neck bifurcation aneurysms such as those of the tip of the BA. In the present study, we evaluated the usefulness of silent MRA compared with that of 3D TOF-MRA for follow-up after Y-configuration stent-assisted coil embolization for basilar tip aneurysms. It has been reported that 3D TOF-MRA and CE-MRA have been used after single stent-assisted coil embolization. ${ }^{12,13,15-17}$ Irie et $\mathrm{al}^{20}$ reported follow-up after stent-assisted coil embolization by using silent MRA, and Cho et $\mathrm{al}^{12}$ reported that the degree of agreement and correlation between $3 \mathrm{D}$ TOF-MRA and 3D rotational angiography was high. With respect to the status of neck remnants, the sensitivity was $80 \%$ ( $4 / 5$ cases). It is thought that magnetic susceptibility artifacts and radiofrequency shielding were decreased because of the very small voxel size (reconstructed voxel size, $0.24 / 0.24 / 0.7 \mathrm{~mm}$ ). Cho et al ${ }^{13}$ reported comparisons between the 3D TOF-MRA and conventional angiography, showing that the MIP plus source images had almost perfect agreement $(\kappa=0.892)$, with better agreement than with the MIP images alone $(\kappa=0.598)$. It is difficult, through the evaluation of source images, to assess the residual flow that is protruding in the direction of the body axis after stent-assisted coil embolization. To confirm the residual flow, MPR with an 


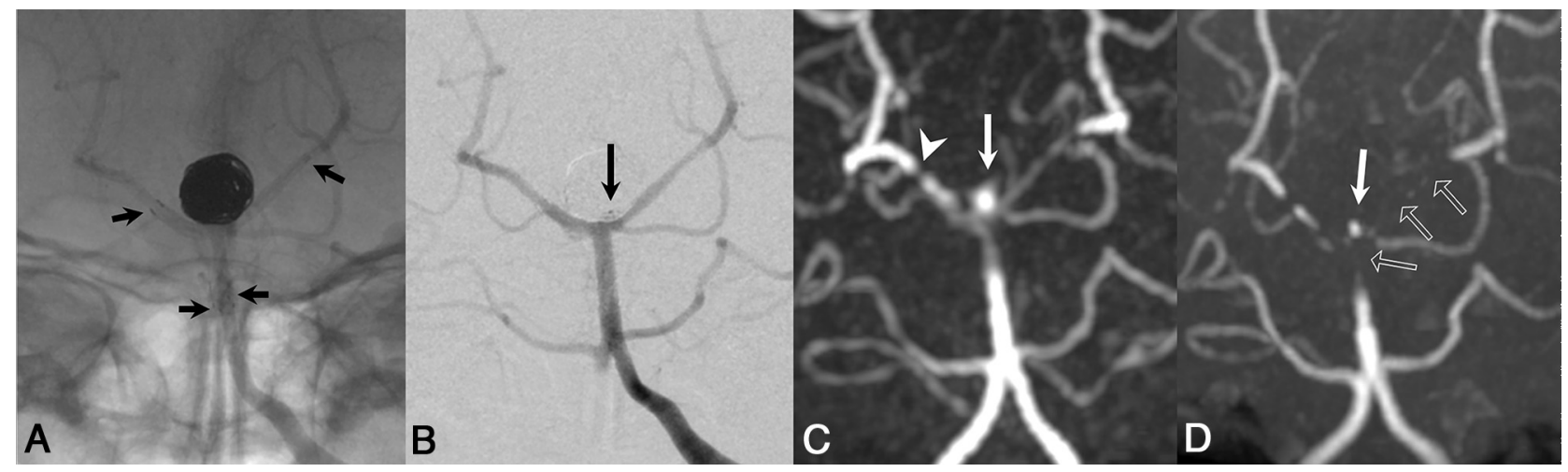

FIG 1. Patient number 1: 53-year-old woman. A, Y-configuration stent-assisted coil embolization is performed with Neuroform (right PCA) and Enterprise stent (left PCA). Short arrows are stent edges. B, X-ray DSA shows neck remnant (long arrow). C, Silent MRA demonstrates minimal signal loss at stented segments; right PCA stent edge segment demonstrates strong signal loss (arrowhead). Neck remnant is depicted (long arrow). D, 3D TOF-MRA on same day shows strong signal loss at BA, and left PCA segment demonstrates complete signal loss (outlined arrows). Neck remnant is depicted (long arrow).
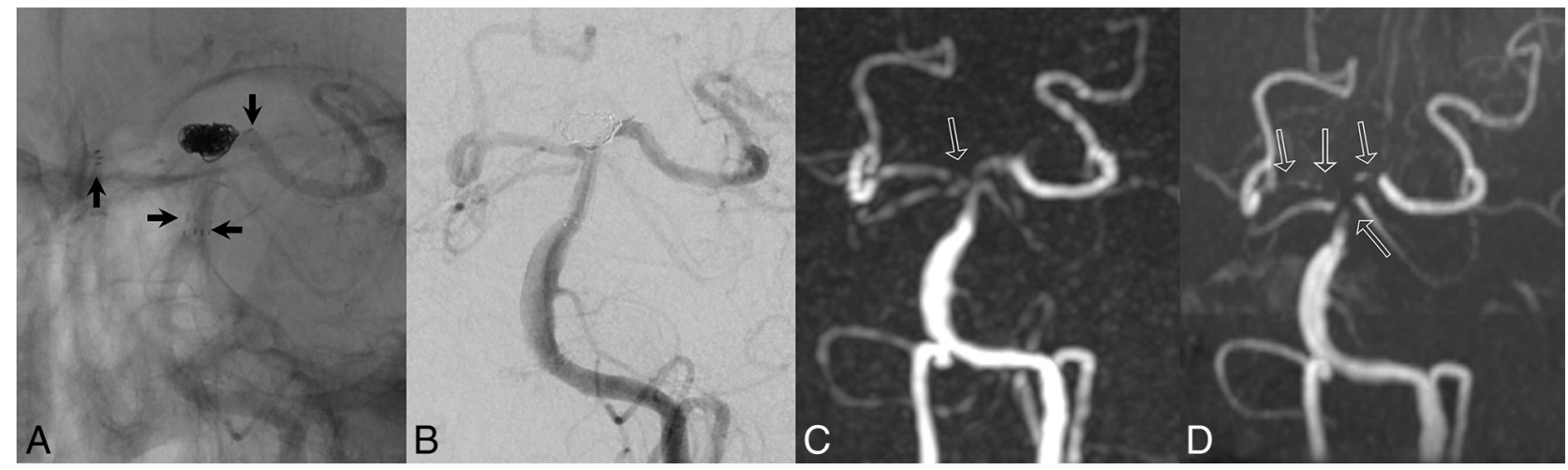

FIG 2. Patient number 2: 75-year-old woman. A, Y-configuration stent-assisted coil embolization is performed with Neuroform (right PCA) and Enterprise stent (left PCA). Short arrows are stent edges. B, X-ray DSA shows complete occlusion. C, Silent MRA demonstrates mild signal loss at right PCA segment. Silent MRA shows complete occlusion. D, 3D TOF-MRA on same day shows mild signal loss at BA, and bilateral PCA segments demonstrates complete signal loss (outlined arrows).
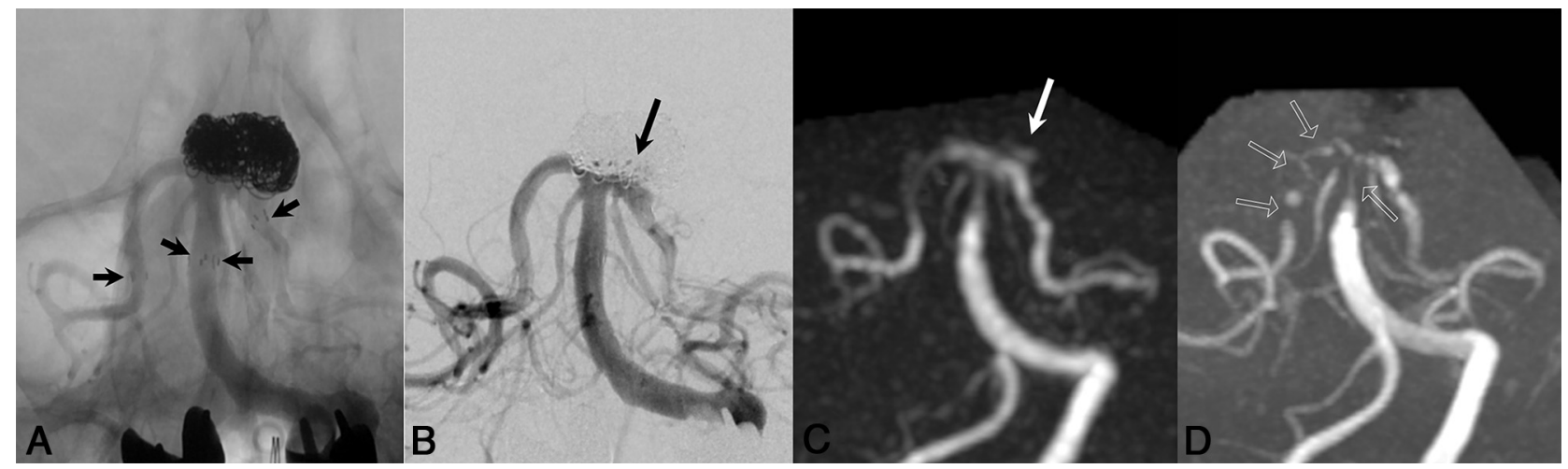

FIG 3. Patient number 3: 66-year-old woman. A, Y-configuration stent-assisted coil embolization is performed with Neuroform (left PCA) and Enterprise stent (right PCA). Short arrows are stent edges. B, X-ray DSA immediately after stent-assisted coil embolization shows neck remnant (long arrow). C, Silent MRA demonstrates minimal signal loss at stented segment and visualizes neck remnant (long arrow). D, 3D TOF-MRA on same day shows mild signal loss at BA, and right PCA segment demonstrates almost complete signal loss (outlined arrows).

accurate image angle should be obtained. Irie et $\mathrm{al}^{20}$ showed the usefulness of the silent MRA for stent-assisted coil embolization rather than a comparison between silent MRA and 3D TOFMRA. Moreover, the visualization of neck remnants was good.

CE-MRA has a better depiction of the flow in a stent than 3D TOF-MRA, and it is reported that CE-MRA has a visualization ability similar to DSA. ${ }^{15}$ Previous reports have examined 3D
TOF-MRA and CE-MRA for stent-assisted coil embolization by using 1 stent; however, a Y-configuration stent-assisted coil embolization case was not examined. Moreover, there have been no studies on the use of MRA for follow-up after Y-configuration stent-assisted coil embolization. The Y stent has a strong influence on magnetic susceptibility artifacts and radiofrequency shielding effects, particularly at the cross point of the 2 stents. It is thought 
that the depiction of the flow in $\mathrm{Y}$ stents is difficult in $3 \mathrm{D}$ TOF-MRA.

On the other hand, CE-MRA has certain problems such as nephrogenic systemic fibrosis, gadolinium accumulation, and anaphylactic shock, and it is not necessarily suitable for repeated examination. Therefore, we examined the usefulness of silent MRA in the present study. Silent MRA is a non-contrast-enhanced MRA technique as is 3D TOF-MRA. In the present study, the visualization ability of silent MRA was higher than that of 3D TOF-MRA for Y-configuration stent-assisted coil embolization. The reason silent MRA is superior in the depiction of the flow in a stent is the data acquisition by UTE. The TE of silent MRA was $0.016 \mathrm{~ms}$ and that of 3D TOF-MRA was $2.9 \mathrm{~ms}$. Gönner et $\mathrm{al}^{21}$ reported that 3D TOF-MRA using UTE reduced the artifacts after coil or stent placement; however, a TE of $2.4 \mathrm{~ms}$ was used in that study. In addition, Yamada et $\mathrm{al}^{22}$ reported that a TE of 1.54-1.60 ms was used in their 3D TOF-MRA study. In silent MRA, UTE can minimize the phase dispersion of the labeled blood flow signal in the voxel and decrease magnetic susceptibility artifacts, and accordingly, the artifacts from stents or coils are decreased.

The Y stent deploys 2 stents from the BA to the bilateral PCA. Moreover, the PCA is positioned almost perpendicular to the BA by stent deployment. Therefore, the PCA has weak inflow effect parallel to a section direction. The visualization ability of the PCA decreased because 3D TOF-MRA was used inflow effect. The limitation of 3D TOF-MRA is spin dephasing by slow flow, turbulent flow, and horizontal directional flow. Moreover, the visualization ability of the BA trunk is worse because of the susceptibility artifacts with 2 stents.

On the other hand, the visualization ability of the BA and PCA is good in silent MRA. The arterial spin-labeling technique is used as a preparation pulse for the visualization of the blood flow. Therefore, visualization of the blood flow does not depend on the flow direction, such as with 3D TOF-MRA in the silent MRA.

In the series, we experienced an interesting case (Fig 1) that used both a Neuroform and Enterprise stent. The Neuroform stent was deployed from the BA trunk to the right PCA, and the Enterprise stent was deployed from the BA trunk to the left PCA. The stent sizes were $3.0 \times 20 \mathrm{~mm}$ and $4.5 \times 22 \mathrm{~mm}$, respectively. In this particular case, the depiction of the distal stent edge of the right PCA showed strong signal loss in both MRAs. Neuroform and Enterprise stents are nitinol stents. ${ }^{12,16,17,23}$ The Neuroform stent has an open-cell design (ie, stent-strut is not interconnected). On the other hand, the Enterprise stent has a closed-cell design (ie, stent-strut is interconnected). Open cell-design stents have lower susceptibility artifacts than closed-cell designs. ${ }^{16,17,23}$ Therefore, the Neuroform stent has a better visualization ability compared with the Enterprise stent.

The marker of Neuroform comprises stainless steel and platinum. ${ }^{15,23}$ On the other hand, the marker of Enterprise comprises tantalum. ${ }^{12,16}$ Agid et $\mathrm{al}^{15}$ reported a "marker band effect" that appeared in small arteries with a diameter of $2 \mathrm{~mm}$ or less and markers of different alloy than the rest of the stent (usually made of platinum), which creates a stronger local susceptibility artifact. ${ }^{15}$ The markers of Neuroform stents are made of platinum, which creates a strong magnetic susceptibility artifact. ${ }^{15}$

In our study, this effect appeared in the right PCA, where the
Neuroform stent (marker made of stainless steel and platinum) with a $3-\mathrm{mm}$ diameter (the smallest diameter in our 7 cases) was deployed. This phenomenon corresponded with that in the report by Agid et $\mathrm{al}^{15}$; it is thought that a similar phenomenon is caused in the silent MRA.

Case number 3 (Fig 3) also used both a Neuroform and Enterprise stent. The left PCA segment was depicted more clearly than the right PCA segment. The Enterprise stent was deployed in the right PCA. Choi et $\mathrm{al}^{23}$ reported that the open-cell design and thinner strut of the Neuroform stent provide better image quality than the Enterprise stent when using 3D TOF-MRA. In the present study, silent MRA more clearly depicted the Neuroform stent than the Enterprise stent, similar to the previous study. The cell design and thickness of the stent strut are important factors in both silent MRA and 3D TOF-MRA.

The depiction of the neck remnants in silent MRA was similar to that of DSA in the present study. Cho et $\mathrm{al}^{13}$ and Agid et al ${ }^{15}$ reported good depiction of the neck remnants in 3D TOF-MRA and CE-MRA in a case of single stent-assisted coil embolization. In the present study, 3D TOF-MRA had poor image quality compared with that reported in previous studies. ${ }^{12,13} \mathrm{With}$ respect to Y-configuration stent-assisted coil embolization, the aneurysm is sandwiched between 2 stents.

Therefore, the influence of 2 stents on the susceptibility artifact was stronger. On the other hand, silent MRA uses UTE and the arterial spin-labeling technique, decreasing susceptibility artifacts and depicting the neck remnants in comparison with $3 \mathrm{D}$ TOF-MRA.

As a similar sequence to silent MRA, Iryo et $\mathrm{al}^{24}$ reported the contrast inherent inflow-enhanced multiphase angiography, or CINEMA, sequence. The CINEMA sequence is a non-contrastenhanced MRA using arterial spin-labeling, such as silent MRA. This is a $4 \mathrm{D}$ MRA sequence. They evaluated patients with AVF. It is thought that the reduction of the susceptibility artifacts can possibly decrease if we shorten TE by this sequence. However, TE of silent MRA is $0.016 \mathrm{~ms}$. TE of CINEMA cannot use UTE, such as silent MRA. They reported that a TE of $2.2 \mathrm{~ms}$ was used. Therefore, it is thought that silent MRA has a higher visualization ability of the artery treated with intracranial stenting compared with the CINEMA sequence. On the other hand, CINEMA has high temporal resolution; as a result, it is useful for a patient needing temporal resolution, such as one with shunt lesions.

On the other hand, Koktzoglou et $\mathrm{al}^{25}$ reported the pointwise encoding time reduction with radial acquisition, or PETRA, sequence. PETRA uses the UTE sequence. ${ }^{26}$ They evaluated carotid stenosis by using a vascular phantom. They reported that arterial spin-labeling MRA could improve the display of hemodynamically significant carotid arterial stenosis. Silent MRA and PETRA MRA are arterial spin-labeling with a UTE MRA sequence. If we use the PETRA MRA sequence, a result similar to that of our examination may be provided.

A major limitation of the present study is the small sample size. The examination of more cases and a variety of stents, lengths, and diameters is required. In the future, we should evaluate stentspecific visualization. There is a report that CE-MRA is useful, ${ }^{15}$ and comparative evaluations with CE-MRA are also required. It is important to elucidate whether silent MRA is not inferior to CE- 
MRA; however, patients in the current study did not undergo CE-MRA. In the current study, silent MRA picked up all neck remnants, with 1 false-positive test result. Thus, we postulate that silent MRA is noninferior to CE-MRA, which needs to be confirmed in future studies.

\section{CONCLUSIONS}

Silent MRA could visualize flow in the stent more clearly than 3D TOF-MRA at the Y-configuration stent-assisted coil embolization for basilar tip aneurysms. Moreover, it is not necessary to use contrast materials. Silent MRA might be useful for follow-up after Y-configuration stent-assisted coil embolization for basilar tip aneurysms.

\section{ACKNOWLEDGMENTS}

The authors thank Shuji Sato, Hideo Kawasaki, and Haruyoshi Hoshito (Radiologic Technologist of the Department of Radiology) for technical advice.

Disclosures: Nao Takano-RELATED: Grant: Cabinet Office of the Government of Japan, * The Ministry of Education, Culture, Sports, Science and Technology of Japan,* Comments: this work was funded by ImPACT Program of Council for Science, Technology and Innovation (Cabinet Office, Government of Japan). This work is supported by a Grant-in-Aid for Scientific Research on Innovative Areas (ABiS) from the Ministry of Education, Culture, Sports, Science and Technology of Japan*; UNRELATED: Payment for Lectures (including service on Speakers Bureaus): GE Healthcare Japan, Comments: 42nd Japanese Society of Radiological Technology Autumn Scientific Congress, October 9-11, 2014; Sapporo, Hokkaido, Japan and luncheon seminar ["Experience of MR angiography (Silent MRA) using Silenz sequence"]. Shigeki Aoki-UNRELATED: Payment for Lectures (including service on Speakers Bureaus): GE Healthcare Japan, Comments: luncheon seminar. *Money paid to the institution.

\section{REFERENCES}

1. Conrad MD, Brasiliense LB, Richie AN, et al. Y stenting assisted coiling using a new low profile visible intraluminal support device for wide necked basilar tip aneurysms: a technical report. J Neurointerv Surg 2014;6:296-300 CrossRef Medline

2. Spiotta AM, Lena J, Chaudry MI, et al. Y-stenting for bifurcation aneurysm coil embolization: what is the risk? Stroke Res Treat 2014; 2014:762389 CrossRef Medline

3. Jeon P, Kim BM, Kim DJ, et al. Y-configuration double-stentassisted coiling using two closed-cell stents for wide-neck basilar tip aneurysms. Acta Neurochir (Wien) 2014;156:1677-86 CrossRef Medline

4. Kono K, Shintani A, Terada T. Retreatment of recanalized aneurysms after Y-stent-assisted coil embolization with double Enterprise stents: case report and systematic review of the literature. Turk Neurosurg 2014;24:593-97 CrossRef Medline

5. Fargen KM, Mocco J, Neal D, et al. A multicenter study of stentassisted coiling of cerebral aneurysms with a Y configuration. $\mathrm{Neu}$ rosurgery 2013;73:466-72 CrossRef Medline

6. Spiotta AM, Gupta R, Fiorella D, et al. Mid-term results of endovascular coiling of wide-necked aneurysms using double stents in a $\mathrm{Y}$ configuration. Neurosurgery 2011;69:421-29 CrossRef Medline

7. Ferns SP, Sprengers ME, van Rooij WJ, et al. Coiling of intracranial aneurysms: a systematic review on initial occlusion and reopening and retreatment rates. Stroke 2009;40:e523-29 CrossRef Medline

8. Kanaan H, Jankowitz B, Aleu A, et al. In-stent thrombosis and stenosis after neck-remodeling device-assisted coil embolization of intracranial aneurysms. Neurosurgery 2010;67:1523-32; discussion 1532-33 CrossRef Medline

9. Mocco J, Fargen KM, Albuquerque FC, et al. Delayed thrombosis or stenosis following Enterprise-assisted stent-coiling: is it safe? Midterm results of the interstate collaboration of Enterprise stent coiling. Neurosurgery 2011;69:908-13; discussion 913-14 CrossRef Medline

10. Kaufmann, TJ Huston J 3rd, Mandrekar JN, et al. Complications of diagnostic cerebral angiography: evaluation of 19,826 consecutive patients. Radiology 2007;243:812-19 CrossRef Medline

11. Lavoie P, Gariépy JL, Milot G, et al. Residual flow after cerebral aneurysm coil occlusion: diagnostic accuracy of MR angiography. Stroke 2012;43:740-46 CrossRef Medline

12. Cho WS, Kim SS, Lee SJ, et al. The effectiveness of 3T time-of-flight magnetic resonance angiography for follow-up evaluations after the stent-assisted coil embolization of cerebral aneurysms. Acta Radiol 2014;55:604-13 CrossRef Medline

13. Cho YD, Kim KM, Lee WJ, et al. Time-of-flight magnetic resonance angiography for follow-up of coil embolization with Enterprise stent for intracranial aneurysm: usefulness of source images. $\mathrm{Ko}$ rean J Radiol 2014;15:161-68 CrossRef Medline

14. Schaafsma JD, Velthuis BK, Majoie CB, et al. Intracranial aneurysms treated with coil placement: test characteristics of follow-up MR angiography - multicenter study 1. Radiology 2010;256:209-18 CrossRef Medline

15. Agid R, Schaaf M, Farb R. CE-MRA for follow-up of aneurysms post stent-assisted coiling. Interv Neuroradiol 2012;18:275-83 Medline

16. Takayama K, Taoka T, Nakagawa H, et al. Usefulness of contrastenhanced magnetic resonance angiography for follow-up of coil embolization with the Enterprise stent for cerebral aneurysms. J Comput Assist Tomogr 2011;35:568-72 CrossRef Medline

17. Choi JW, Roh HG, Moon WJ, et al. Time-resolved 3D contrastenhanced MRA on 3.0T: a non-invasive follow-up technique after stent-assisted coil embolization of the intracranial aneurysm. $\mathrm{Ko}$ rean J Radiol 2011;12:662-70 CrossRef Medline

18. Kanda $\mathrm{T}$, Ishii $\mathrm{K}$, Kawaguchi $\mathrm{H}$, et al. High signal intensity in the dentate nucleus and globus pallidus on unenhanced T1-weighted MR images: relationship with increasing cumulative dose of a gadolinium-based contrast material. Radiology 2014;270:834-41 CrossRef Medline

19. Alibek S, Vogel M, Sun W, et al. Acoustic noise reduction in MRI using Silent Scan: an initial experience. Diagn Interv Radiol 2014;20: 360-63 CrossRef Medline

20. Irie R, Suzuki M, Yamamoto M, et al. Assessing blood flow in an intracranial stent: a feasibility study of MR angiography using a silent scan after stent-assisted coil embolization for anterior circulation aneurysms. AJNR Am J Neuroradiol 2015;36:967-70 CrossRef Medline

21. Gönner F, Heid O, Remonda L, et al. MR angiography with ultrashort echo time in cerebral aneurysms treated with Guglielmi detachable coils. AJNR Am J Neuroradiol 1998;19:1324-28 Medline

22. Yamada N, Hayashi K, Murao K, et al. Time-of-flight MR angiography targeted to coiled intracranial aneurysms is more sensitive to residual flow than is digital subtraction angiography. AJNR Am J Neuroradiol 2004;25:1154-57 Medline

23. Choi JW, Roh HG, Moon WJ, et al. Optimization of MR parameters of 3D TOF-MRA for various intracranial stents at 3.0T MRI. Neurointervention 2011;6:71-77 CrossRef Medline

24. Iryo $\mathrm{Y}$, Hirai $\mathrm{T}$, Kai $\mathrm{Y}$, et al. Intracranial dural arteriovenous fistulas: evaluation with 3-T four-dimensional MR angiography using arterial spin labeling. Radiology 2014;271:193-99 CrossRef Medline

25. Koktzoglou I, Giri S, Piccini D, et al. Arterial spin labeled carotid MR angiography: a phantom study examining the impact of technical and hemodynamic factors. Magn Reson Med 2016;75:295-301 CrossRef Medline

26. Grodzki DM, Jakob PM, Heismann B. Ultrashort echo time imaging using pointwise encoding time reduction with radial acquisition (PETRA). Magn Reson Med 2012;67:510-18 CrossRef Medline 